\title{
Left ventricular pacing via the coronary sinus in a patient with a mechanical tricuspid valve
}

\author{
Jakub Zawadzki ${ }^{1, \mathrm{D}}$, Łukasz Januszkiewicz ${ }^{1, \mathrm{~B}, \mathrm{E}}$, Andrzej Cacko ${ }^{1, \mathrm{~B}}$, Marcin Michalak ${ }^{1, \mathrm{~B}, \mathrm{E}}$, Grzegorz Opolski1, ${ }^{1, \mathrm{~B}}$, \\ Marcin Grabowski ${ }^{1, \mathrm{~A}-\mathrm{B}, \mathrm{E}}$
}

A - Research concept and design, B - Collection and/or assembly of data, C - Data analysis and interpretation,

D - Writing the article, E - Critical revision of the article, F - Final approval of article

1. 1st Department of Cardiology, Medical University of Warsaw

Address for correspondence:

Jakub Zawadzki, 1st Department of Cardiology, Medical University of Warsaw email: zawadzkijakub@o2.pl

Łukasz Januszkiewicz, 1st Department of Cardiology, Medical University of Warsaw

email: lukasz.januszkiewicz@wum.edu.pl

Andrzej Cacko, 1st Department of Cardiology, Medical University of Warsaw email: andrzej.cacko@wum.edu.pl

Marcin Michalak, 1st Department of Cardiology, Medical University of Warsaw email: marcin.michalak@wum.edu.pl

Grzegorz Opolski, 1st Department of Cardiology, Medical University of Warsaw

email: grzegorz.opolski@wum.edu.pl

Marcin Grabowski, 1st Department of Cardiology, Medical University of Warsaw

email: marcin.grabowski@wum.edu.pl

Received: 09.08.2017

Revised: 17.12 .2017

Accepted: 17.12.2017

Final review: 28.11.2017

DOI: $10.24255 / \mathrm{hbj} / 81266$

\section{Key words:}

\section{pacemaker, ventricular pacing, prosthetic valve}

\section{Abstract}

A 56-year-old man was referred to the clinic for implantation of a pacemaker due to symptomatic junctional bradycardia. His medical history was positive for Starr-Edwards ball tricuspid valve implantation, excision of a right ventricular

\section{Case}

A 56-year-old man was referred for implantation of a pacemaker due to exacerbation of symptomatic junctional bradycardia, which was also observed in the electrocardiogram and confirmed in the intracardiac electrogram (Figure 1). His medical history was positive for Starr-Edwards ball valve implantation in the tricuspid position, excision of a right ventricular myxoma, both in 1979, and reoperation of this area for prosthetic valve thrombosis 37 years prior to the current admission, while being negative for features of heart failure.

Intraoperative ECG revealed a prolonged PR interval, which, in combination with symptomatic bradycardia in sick sinus disease, was an indication for dual chamber pacemaker myxoma and reoperation of this area for thrombosis. Due to previous surgery, we implanted a dual-chamber pacing system with the ventricular lead introduced

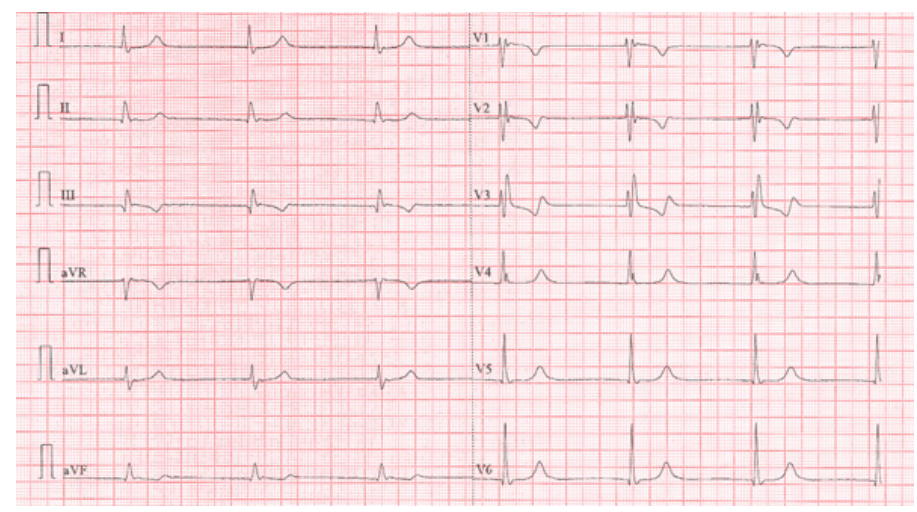


implantation. Right ventricular and epicardial pacing were withdrawn because of the risk of mechanical tricuspid valve impairment and potential adhesions after previous cardiac surgery. First, the outer catheter (Biotronik Selectra Extended Hook) was introduced into the coronary sinus (CS) ostium as shown in Figure 2A. Due to the enlarged right atrium, to obtain acceptable pacing parameters the atrial lead was used and lateral x-ray showed its separation from the coronary sinus lead. Then a Quartet Quadripolar LV Lead was implanted into the anterior coronary vein (Figure $2 \mathrm{~B}$ ). Acute measurements of pacing parameters were: threshold $1.0 \mathrm{~V}$ at $0.4 \mathrm{~ms}$, $\mathrm{R}$ wave $4.9 \mathrm{mV}$, impedance $890 \mathrm{ohms}$ at $2.98 \mathrm{~V}$.

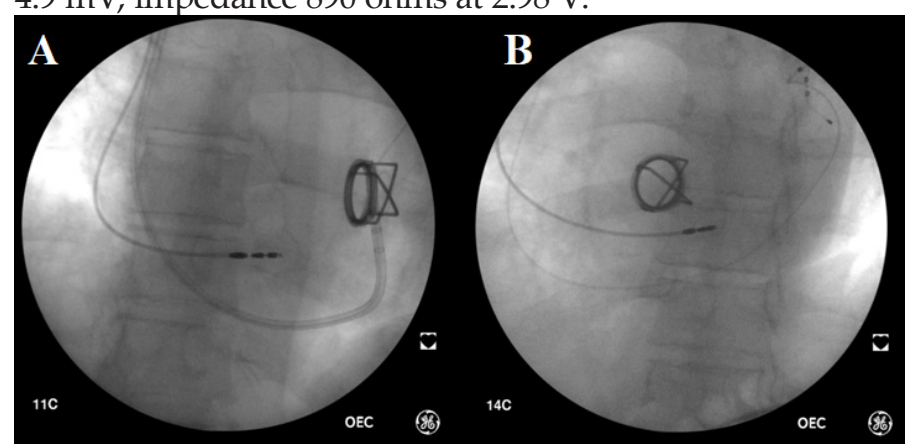

Figure 2. Fluoroscopic right anterior intra-operative and left anterior oblique post-implantation projections.

The procedure was uncomplicated. During the several-day follow-up, leads' electrical parameters were stable and the function of the mechanical valve and the pacing system was normal. (Figure 3)

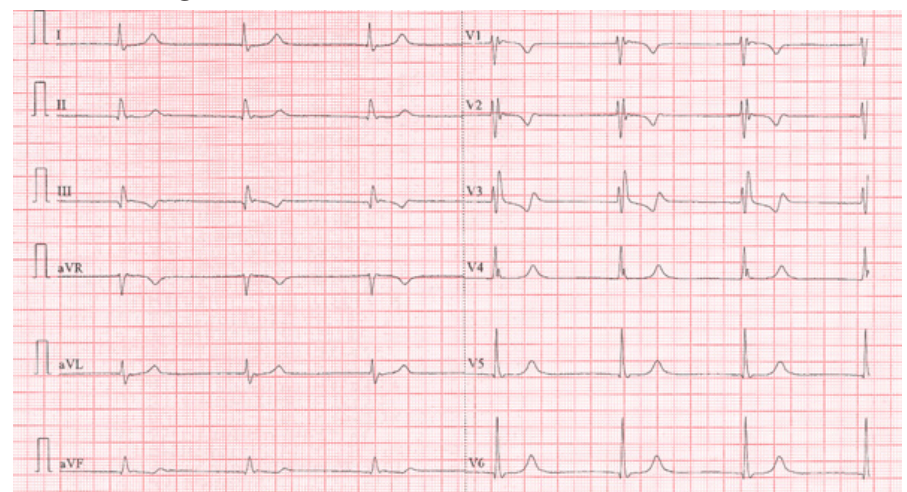

Figure 3. Electrocardiography at the standard chart speed of $25 \mathrm{~mm} / \mathrm{s}$, after implantation of a dual chamber pacemaker with left ventricular pacing features: right heart axis, dominant $\mathrm{R}$ wave in V1 and right bundle branch block. Arrows indicate spikes for atrial pacing.

\section{Discussion}

Pacing and defibrillation therapy in tricuspid mechanical valve recipients remains challenging. In cases of sinus bradycardia, only atrial pacing can be used. However, in our patient presence of first-degree atrioventricular block with a right bundle branch block-like pattern might have resulted in a need for ventricular pacing in future, with the risk that use of atrial pacing only may require future upgrading to dual-chamber pacing. Upgrades with additional lead implantation are associated with a notable complication risk. These data support our decision to provide ventricular pacing in this case. ${ }^{[1]}$

In the present case, due to previous surgery and the possibility of adhesion, access for right ventricular epicardial pacing was limited. Moreover, epicardial pacing is usually associated with development of a high threshold and requires limited thoracotomy ${ }^{[2]}$; therefore it remained the second choice. We implanted a dual-chamber pacing system with the ventricular lead introduced through the CS. Due to the lack of resynchronization recommendations to use the lateral or posterolateral side branch of the CS, the lead was placed in the anterior branch, which was assessed by the implanter as a stable location with minimal probability of displacement. The use of ventricular pacing through the CS in patients with a prosthetic tricuspid valve seems to provide effective pacing and sensing measurements, with a minimally invasive approach, while taking precautions due to the proximity of the coronary sinus ostium to the right atrioventricular valve. This method was first described by Bai et al. in $1994^{[3]}$; several authors have used the method over the years. ${ }^{[4,5,6,7]}$

\section{References}

1. Poole Je, Gleva Mj, Mela T, et al. Complication rates associated with pacemaker or implantable cardioverter-defibrillator generator replacements and upgrade procedures: results from the REPLACE registry. Circulation. 2010 Oct 19;122(16):1553-61.

2. Vijayakumar M, Kamath P, Pai P G. Permanent pacing in a patient with tricuspid prosthesis widening therapeutic use of coronary sinus. Indian Heart J. 2013 Sep; 65(5): 611-613.

3. Bai Y, Strathmore N, Mond H, Grigg L, Hunt D. Permanent ventricular pacing via the great cardiac vein. Pacing Clin Electrophysiol 1994;17:678-83. 7.

4. Grimard C, Clémenty N, Fauchier L, Babuty D. Ventricular pacing through coronary sinus in patients with tricuspid prosthesis. Ann Thorac Surg. 2010 Jun;89(6):e51-2.

5. Ząbek A, Małecka B, Tomala I, Matusik P, Boczar K, Lelakowski J. Cardiac pacing in a patient with mechanical tricuspid valve. Pol Arch Med Wewn. 2015; 125 (1-2): 89-91.

6. Yoda M, Nakai T, Okubo K, Hata M. First case report in Japan of left ventricular pacing via a coronary vein in a patient with a mechanical tricuspid valve. Circ J 2008; 72 : 335 - 336 .

7. Lopez JA, Lufschanowski R. A novel approach to transvenous dual-chamber pacing lead placement and cardiac defibrillator implantation after tricuspid valve replacement. J Cardiovasc Electrophysiol. 2008 Aug;19(8):873-5. 